\title{
Massive ascites mimicking acute urinary retention
}

\author{
Sheng-Huei Wang ${ }^{1} \cdot$ Kuang-Yu Wei ${ }^{1}$ Kuan-Yu Chen ${ }^{1} \cdot$ Wei-Lin Lin $^{3}$. \\ Wei-Shiang Lin $^{2}$
}

Received: 10 December 2015/ Accepted: 19 December 2015/Published online: 9 January 2016

(C) SIMI 2016

A 48-year-old man with a history of dilated cardiomyopathy was admitted because of acute decompensated heart failure. On day 4 , the patient was transferred to the intensive care unit because of shock of unknown etiology. The patient complained of lower abdominal fullness, and the urine output was only $40 \mathrm{ml}$ in the preceding $10 \mathrm{~h}$. Suprapubic abdominal ultrasonography showed massive fluid accumulation (Fig. 1a, b). With the impression of acute urine retention, urethral catheterization was performed without difficulty, but no urine drainage occurred. A urethral false passage was suspected, and Urology was consulted for cystoscopy. Before cystoscopy, the patient had an abdominal computed tomography that showed massive ascites and an empty bladder (Fig. 1c). Heart failure-related massive ascites and prerenal acute kidney

Wei-Shiang Lin

wslin545@ms27.hinet.net

1 Department of Internal Medicine, Tri-Service General Hospital, National Defense Medical Center, Taipei, Taiwan, Republic of China

2 Division of Cardiology, Department of Internal Medicine, Tri-Service General Hospital, National Defense Medical Center, Taipei, Taiwan, Republic of China

3 Department of Anesthesiology, Tri-Service General Hospital, National Defense Medical Center, Taipei, Taiwan, Republic of China injury due to diuretic overdose over several days were diagnosed.

Excluding post-renal acute kidney injury is the first of diagnosis in a patient with limited urine output and abdominal fullness. Although we performed ultrasonography on the patient, two factors led to misdiagnosis initially. First, an unskilled practitioner misinterpreted the ascites as a large bladder on ultrasonic imaging. Second, right-sided heart failure is a less common cause of massive ascites than cirrhosis in clinical practice.

In the present case, pelvic ascites mimicked urine in the bladder, and a urethral false passage was suspected because the Foley catheter balloon was outside the fluid. A full bladder is usually rectangular, thin-walled in configuration, and anechoic with the urine on a transverse scan [1]. In comparison, the border confining the ascites in this case was pear-shaped and hyperechoic. The hyperechoic reverberation in the fluid on ultrasonography was the empty bladder (Fig. 1a). Ultrasonography of Morrison's pouch or the splenorenal recess for ascites detection is another technique for a differential diagnosis. Skillfully interpreted abdominal ultrasonography prevented unnecessary invasive procedures in the present case. 
Fig. 1 a Transverse view of the suprapubic abdominal ultrasonogram shows abundant fluid accumulation and hyperechoic reverberation in the fluid (arrow). b Sagittal view of the ultrasonogram shows the Foley catheter balloon outside the accumulated fluid (arrow) c Abdominal computed tomography shows massive ascites in the peritoneal cavity. The pelvic ascites mimics a distended bladder, and a Foley catheter balloon is seen within the empty bladder

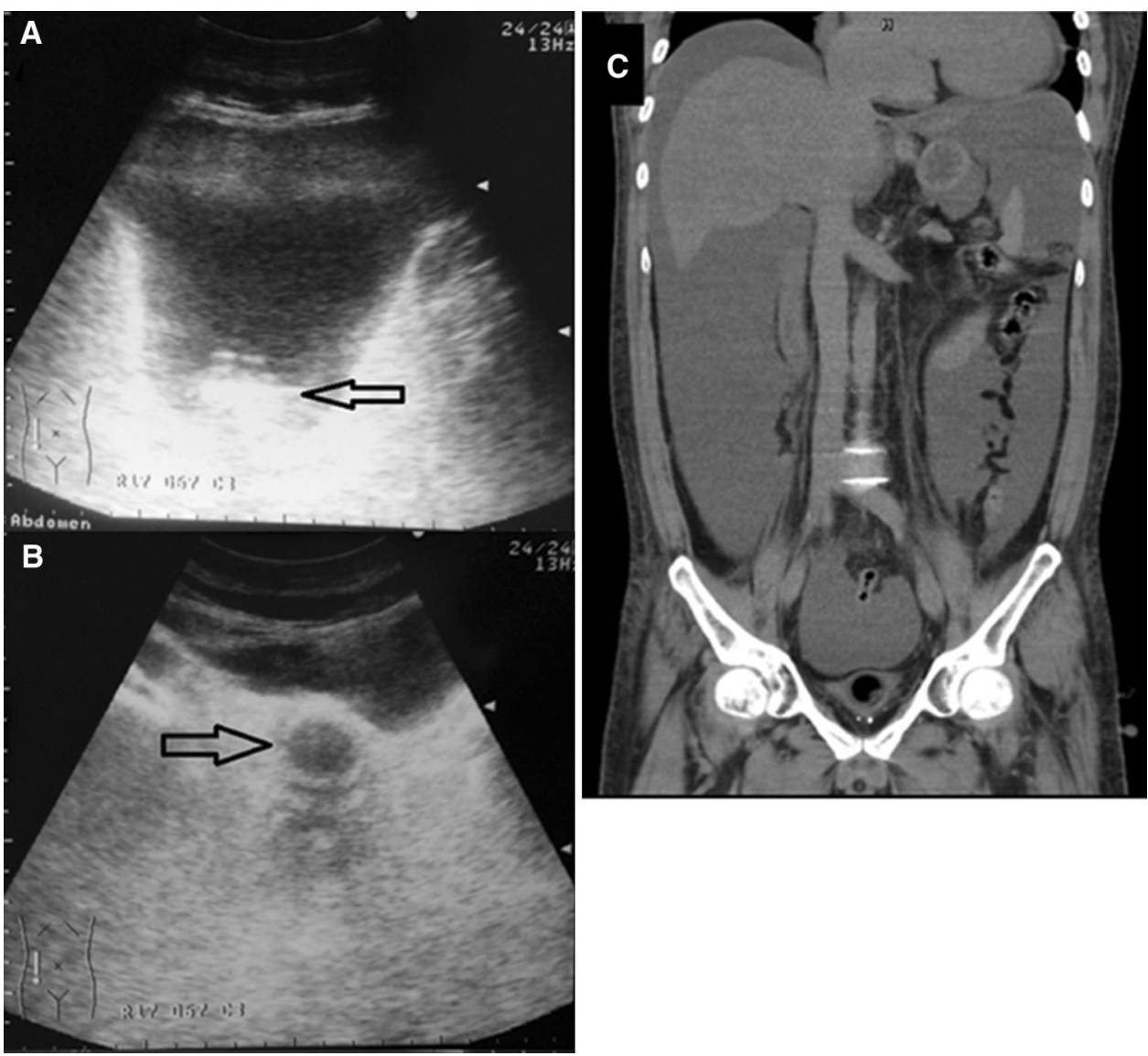

\section{Compliance with ethical standards}

Conflict of interest The authors declare that they have no conflict of interest.

Statement of human and animal rights The authors hereby declare that the research documented in the present manuscript, involving human participant, has been carried out in accordance with the ethical standards of the institutional and national research committee and have been performed in accordance with the ethical standards laid down in the 1964 Declaration of Helsinki and its later amendment or comparable ethical standards.
Informed consent Written informed consent was obtained from the patient.

\section{Reference}

1. Bala KG, Chou YH (2010) Ultrasonography of the urinary bladder. J Med Ultrasound 18:105-114 